\title{
Retraction Note to: Structure Vibration Analysis by Active Noise Control of Power Transformer
}

\author{
Young-Dal Kim ${ }^{1}$, Jae-Myung Shim ${ }^{1}$, Keun-Seok Park ${ }^{1}$, Yun-Mi Jeong ${ }^{1}$, \\ and Dae-Dong Lee ${ }^{2}$ \\ ${ }^{1}$ Department of Electrical Engineering, Hanbat National University, \\ Daejeon, Republic of Korea \\ \{zeromoon, jmshim, jym0809\} @hanbat.ac.kr, \\ smg-park@nate.com \\ ${ }^{2}$ Department of Electrical Engineering, Hanyang University, \\ Seoul, Republic of Korea \\ ldd77@hanbat.ac.kr
}

\section{Retraction Note to:}

Chapter "Structure Vibration Analysis by Active Noise Control of Power Transformer" in: T.-h. Kim et al. (Eds.):

Computer Applications for Security, Control and System Engineering, CCIS, DOI: 10.1007/978-3-642-35264-5_32

The paper starting on page 234 of this volume has been retracted due to multiple publication. 